\title{
Glioma-Associated Oncogene Homolog1 (Gli1)-Aquaporin1 pathway promotes glioma cell metastasis
}

\author{
Zheng-qiang Liao ${ }^{1, \#}$, Ming Ye ${ }^{2, \#}$, Pei-gen $\mathrm{Yu}^{1}$, Chun Xiao ${ }^{1}$ \& Feng-yun Lin $^{3, *}$ \\ ${ }^{1}$ Department of Neurosurgery, The Nine People's Hospital of Chongqing, Chongqing, ${ }^{2}$ Department of Neurosurgery, The First Affiliated \\ Hospital of Suzhou University, Suzhou, ${ }^{3}$ Department of Pharmaceutics, Chongqing Medical and Pharmaceutical College, Chongqing, China
}

\begin{abstract}
Glioma-Associated Oncogene Homolog1 (Gli1) is known to be activated in malignant glioma; however, its downstream pathway has not been fully explained. The aim of this study was to explore the role of Gli1-Aquaporin1 (AQP1) signal pathway in glioma cell survival. Our data suggests that both Gli1 and AQP1 are upregulated in glioma tissues, as in comparison to in normal tissues. These up-regulation phenomena were also observed in glioma U251 and U87 cells. It was demonstrated that Gli1 positively regulated the AQP1 expression. By luciferase reporter gene and ChIP assay, we observed that this modulation process was realized by combination of Gli1 with AQP1 promotor. In addition, knock down of Gli1 by siRNA interference reduced the viability of glioma cells as well as suppressed cell metastasis. Also, the inhibitory effects of cell survival by silenced Gli1 were abrogated by AQP1 overexpression. In summary, glioma cell survival is a regulatory process and can be mediated by Gli1-AQP1 pathway. [BMB Reports 2016; 49(7): 394-399]
\end{abstract}

\section{INTRODUCTION}

Malignant gliomas are primary brain cancers arising from the glial cells (1) located in the central nervous system (CNS). These glial cells play pivotal roles in the maintenance of nerve cell function. Gliomas have been recognized as the leading cancer killers, with an average life expectancy of less than 15 months, due to its aggressive and high recurrence rate (2). In the clinical setting, the preferred mode of treatment is excision, followed by radiation or chemotherapy. However, surgery is unable to remove all glial tumors, and the residual tumor can invade adjacent normal tissue, subsequently to high

${ }^{*}$ Corresponding author. Tel: +86-023-68862495; Fax: +86-02361691266; E-mail: lin fengyun54@163.com

${ }^{\#}$ These authors contributed equally to this work.

http://dx.doi.org/10.5483/BMBRep.2016.49.7.011

Received 18 January 2016, Revised 16 February 2016, Accepted 26 April 2016

Keywords: Invasion, Migration, U251, U87 recurrences (3). In view of this, metastasis blockade might be an effective therapeutic pathway for gliomas.

Cancer results from internal adjustment disorders in response to various stimuli. Recently, accumulated evidence suggested that glioma cell metastasis is a highly regulated process $(4,5)$, which is characterized by the deregulation of many key signaling pathways involving growth, proliferation, survival, and apoptosis. Glioma-associated Oncogene Homolog 1 (Gli1) was initially named in glioma research, and its product Gli1 is a transcriptional factor that functions as an effector of the Hedgehog $(\mathrm{Hh})$ signaling pathway (6). Hh-Gli1 pathway was found to be disordered and abnormally activated in gliomas. For functional regulation, the Gli1 transcription factors activate/inhibit transcription by binding to Gli1 responsive genes and by interacting with the transcription complex. For example, Gli1 can affect glioma apoptosis and proliferation of glioma cell via regulated CyclinD1 and $\mathrm{Bcl}-2$ (7), which is essential for glioma migration and invasion.

Notably, down-regulated Gli1 was proved to be associated with organic edema in damaged brain (8) or chronic pancreatitis (9). Also, previous studies have revealed a possible correlation between glioma metastasis and the composition of the peritumoral edema (10). Based on these data, Gli1 may play a regulatory role in water balance of glioma. Thus, we were to explore the regulated role of Gli1 on Aquaporin1 (AQP1) expression. Aquaporin1 (AQP1) belongs to the small molecule channel protein family and commonly exists in the glioma cell membrane. AQP1 can effectively and rapidly transport water molecules transmembrane. Recent research has pointed out that AQP1 is associated in the development of nervous system disease, especially in tumor pathogenesis (11, 12). Upregulation of AQP1 contributes to enhancement of the invasive potential of glioma cells $(12,13)$. Taking this into account, the expressional regulation of AQP1 might be beneficial for glioma treatment. However, the regulatory mechanism of AQP1 expression in glioma remains unclear.

In this study, we sought to determine whether the Gli1-AQP1 pathway is involved in glioma metastasis. We first examined the expression of Gli1 and AQP1 in advanced glioma tissue and in normal tissues. Subsequently, we evaluated the possible regulatory role of Gli1 on AQP expression, as well as explored their role in glioma cell

ISSN: 1976-670X (electronic edition)

Copyright (C) 2016 by the The Korean Society for Biochemistry and Molecular Biology

c) This is an open-access article distributed under the terms of the Creative Commons Attribution Non-Commercial License (http://creativecommons.org/licenses/by-nc/4.0) which permits unrestricted non-commercial use, distribution, and reproduction in any medium, provided the original work is properly cited. 
metastasis in two glioma cell lines of U251 and U87 in vitro.

\section{RESULTS}

Expression of Gli1 and AQP1 in glioma tissue and glioma cell Abnormal expressions of Gli1 and AQP1 in 10 malignant glioma tissues were compared to 3 normal tissues. By qRT-PCR and western blotting, we found that both Gli1 and AQP1 were significantly upregulated in in glioma tissues compared with normal tissues in respect of mRNA and protein level (Fig. 1A). The elevated levels of AQP1 mRNA and Gli1 mRNA were positively correlated (Fig. 1B).

To investigate a putative role for Gli1 in glioma, we firstly determined the expression of Gli1 in two human glioma cell lines, U251 and U87. The result from qRT-PCR analysis showed that Gli1 mRNA was significantly increased compared with normal human astrocytes. The corresponding protein
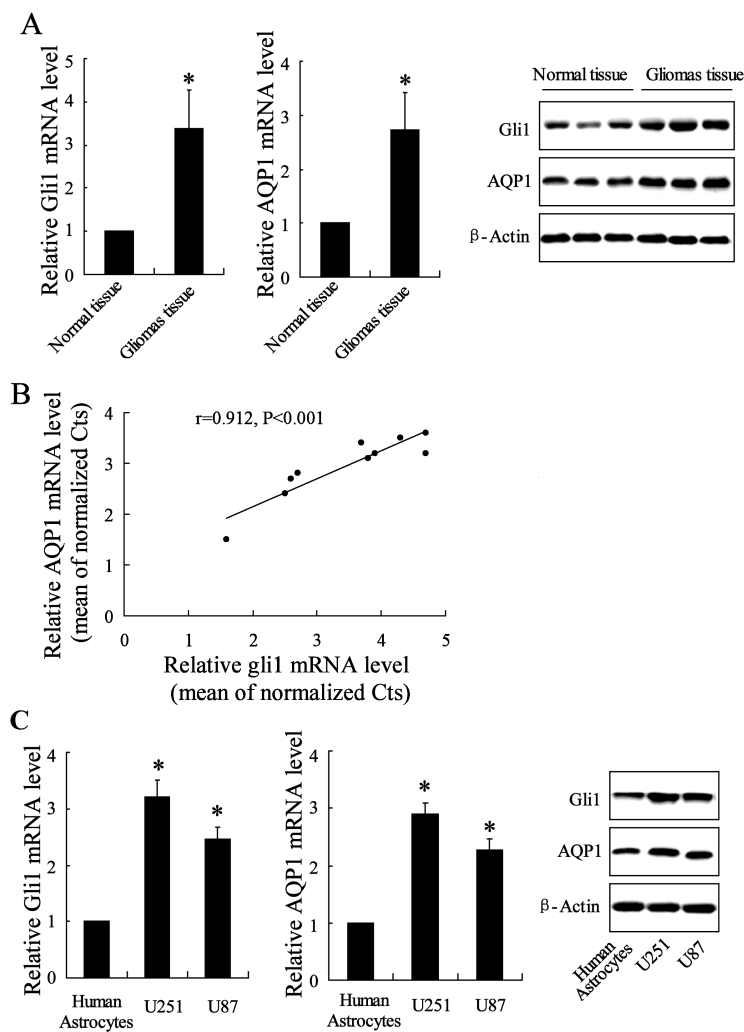

Fig. 1. Expression of Gli1 and AQP1 in glioma tissue and glioma U251, U87 cell lines. (A) Relative mRNAs levels and representative banes of Gli1 and AQP1 in glioma tissues were determined using qRT-PCR and western blotting. (B) Correlation analysis of expression of Gli1 mRNA and AQP1 mRNA. (C) Relative mRNA levels and representative banes of Gli1 and AQP1 were determined in two glioma cell lines. Data are representative of three experiments. Data presented as mean \pm SD. ${ }^{*} \mathrm{P}<0.05$ vs. normal tissue or human astorocytes. expression was next confirmed by western blot assay using a commercial antibody raised against amino acids 781-1080 of Gli1 of human origin. In accordance with observation in glioma tissues, expression of AQP1 in levels of mRNA and protein was also enhanced (Fig. 1C).

\section{Gli1 regulates AQP1 expression by binding to its promotor} Gli1 functions as transcriptional factor and regulated gene expression in response to cellular conditions change. We next explored the role of Gli1 as a potential regulator of AQP1 expression in glioma cell. U251 and U87 were treated with si-Gli1 with empty plasmid vector as si-control, which contributed to substantially downregulated Gli1 mRNA as well as protein. The confirmation of AQP1 gene expressional regulation by Gli1 was based on the analysis of alteration of AQP1 mRNA and protein, which showed an expressional reduction following the knock down of Gli1 (Fig. 2A).

In order to confirm the reduction of AQP1 expression due to knock down of Gli1, we next evaluated AQP1 promotor activity in si-Gli1 (Fig. 2B) or pcDNA-Gli1 (Fig. 2C) transfected U251 and U87 cells. The data showed that activity of Gli1 promotor was significantly reduced by si-Gli1 treatment (Fig. 2B), whereas it was enhanced by Gli1 overexpression (Fig. 2C). The result of ChIP assay for combination of Gli1 and AQP promotor is shown in Fig. 2D. Gli1 antibody can bind to -406- -208 of AQP1 promotor.

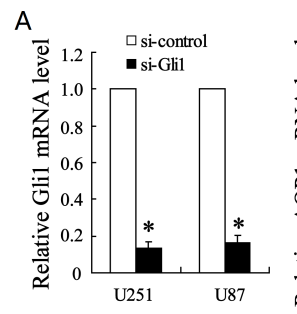

B
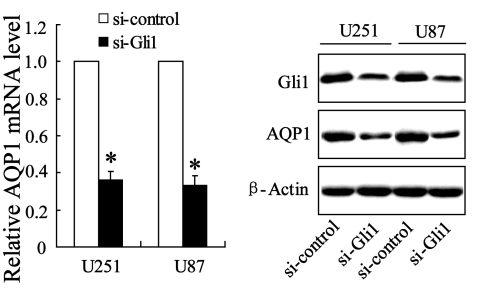

D
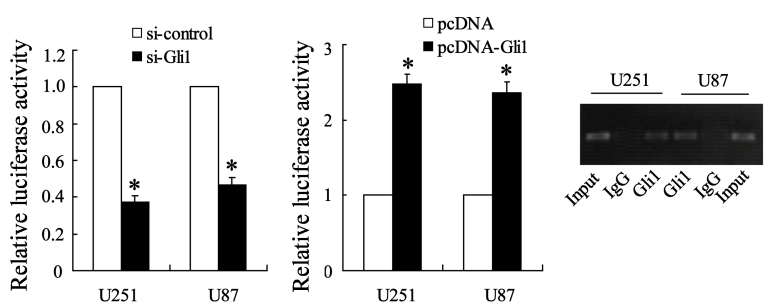

Fig. 2. Gli1 regulates transcriptional activity of AQP1. (A) qRT-PCR or western blot were performed to determine the expression of Gli1 and AQP1 gene expression in si-Gli1-transfected U251 and U87 cells. Luciferase activity of AQP1 was evaluated in (B) cells co-transfected with si-Gli1 and luciferase labelled AQP1 for 48 hours and $(C)$ cells co-transfected with pcDNA-Gli1 and luciferase labelled AQP1 for 48 hours; luciferase activity was normalized to si-control and pcDNA, respectively. (D) The combination of Gli1 on AQP1 promotor was confirmed by ChIP assay. Data presented as mean \pm SD from three different experiments. ${ }^{*} \mathrm{P}<0.05$ vs. control. 
Gli1-AQP1 pathway negatively regulated glioma cell viability In order to examine if inactivated Gli1-AQP1 pathway is essential for glioma cell viability, U251 and U87 were transfected with si-Gli1, a recombinant plasmid containing a specific inference sequence for Gli1. Cell viability was assessed using MTT assay, and the result showed a reduction trend in comparison with si-control. This inhibitory action of si-Gli1 on U251 and U87 cell viability was abrogated by the overexpression of AQP1 in cells co-transfected with pcDNA-AQP1 (Fig. 3A). Co-transfection of si-Gli1 and pcDNA-AQP1 caused upregulation of AQP1 with no corresponding effect of Gli1 expression (Fig. 3B). Moreover, AQP1 knockdown alone also reduced the cell viability (Fig. 3C). These data suggested the negatively regulatory of Gli1-AQP1 pathway in glioma cell viability.

\section{Gli1-AQP1 pathway regulated glioma cell metastasis}

To explore the effect of Gli1-AQP1 axis on glioma cell metastasis, U251 and U87 were treated with si-Gli1 with or without combination of pcDNA-AQP1 with the purpose of evaluating the abilities of cell migration and invasion. From
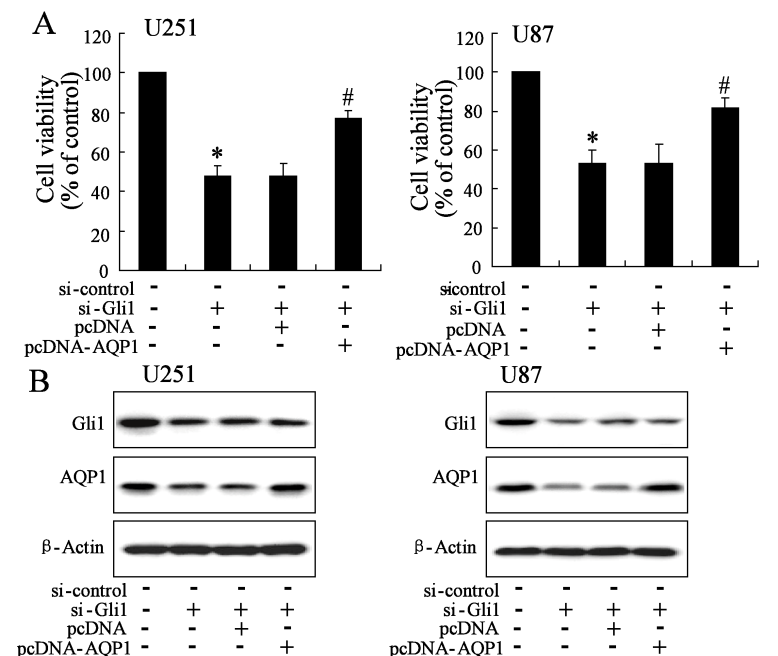

pcDNA-AQP1
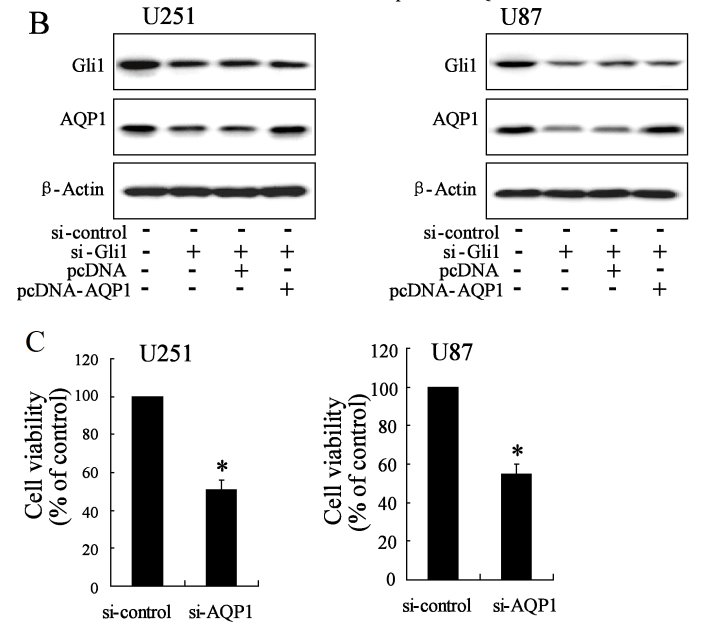

Fig. 3. Regulatory role of Gli1-AQP1 axes on cell viability. (A) MTT assay was performed to determine U251 and U87 cell viability in cells treated with si-Gli1 and/or pcDNA-AQP1. (B) Western bolt presented expression of Gil1 and AQP1. (C) Cell viability was determined in si-AQP1-transfected U251 and U87 cells. Data presented as mean \pm SD from three different experiments. $* P<$ 0.05 vs. control; $\mathrm{\#} P 0.05$ vs. si-Gli $1+$ pcDNA. the picture of migration and invasion, we observed that the migrated and invaded U251 cells (upper of Fig. 4A) and U87 cells (upper of Fig. 4B) were less than in si-control treatment; these inhibitory metastasis actions were reversed by si-Gli1 co-transfected with pcDNA-AQP1. Moreover, this was intuitively demonstrated by direct counting viable cell (lower of Fig. $4 \mathrm{~A}$ and $4 \mathrm{~B})$.

\section{DISCUSSION}

Malignant gliomas often recur within 1-2 $\mathrm{cm}$ of the original tumor site because of the tumor cell invasion into the surrounding normal brain tissue where they can evade surgical removal and radiation therapy. In view of this disadvantage of the current therapeutic method, efforts on exploring the reliable therapeutic targets based on identifying the molecular pathological process of gliomas, might provide a better cure and therapy for glioma (14). In the present study, we confirmed the key role of Gli1-AQP1 signal pathway in glioma cell metastasis and survival.

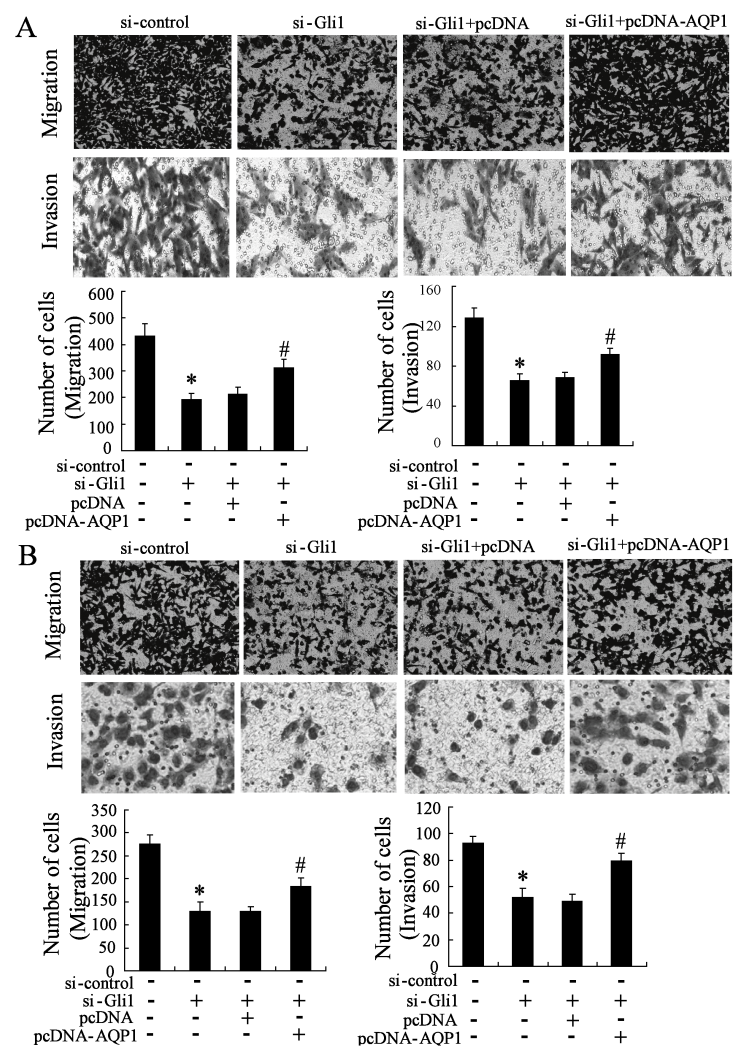

Fig. 4. Regulatory role of Gli1-AQP1 axes on cell metastasis. Cell migration and invasion were examined in (A) U251 and (B) U87 cells treated with si-Gli1 and/or pcDNA-AQP1. Data presented as mean \pm SD from three different experiments. ${ }^{*} P<0.05$ vs. control; ${ }^{\#} \mathrm{P}<0.05$ vs. si-Gli1 + pcDNA. 
Gli1, a full-length transcriptional activator, belongs to the Gli family of Zinc-finger transcription factors and has been shown to be involved in the intracellular signal transduction controlled by the Hedgehog signaling pathway. Also, it functions as a key regulator for important developmental events of the central nervous system. It has previously been shown that the Gli1 variant is expressed in most gliomas and patient-derived xenografts (15), but is undetectable in normal brain cells or tissues. Our present data is similar to the previous studies, and showed the activation of Gli1 expression in glioma tissue as well as in two glioma cell lines (U251 and U87) by qRT-PCR and western blotting analysis. Although a comprehensive analysis of direct transcriptional targets of Gli1 at the genomic level is lacking, a number of studies have shown that Gli1 directly regulates the transcription of various genes that are known to be involved in cell proliferation, survival, and chemotolerance (16-18).

Apart for functioning as a molecule channel protein, AQP1 was recently proved to be involved in various tumorigeneses. AQP1 is regarded as a renal cancer specific protein that can be detected in urine (19). In in vitro lung cancer cell culture, AQP1 knockdown significantly decreases cancer cell migration and metastasis (20). El Hindy et al. found that AQP1 polymorphism serves as a survival prognosticator in patients suffering from glioblastoma-multiforme (11). These data suggested the potential functional role of AQP1 in glioma. More recently, efforts are increasingly being focused on elaborating the regulatory mechanism of AQP1 protein expression. In the present study, we were first prompted to examine the expressional alteration of AQP1 in gliomas cells in accordance with elevated Gli1 levels, and demonstrate this regulatory interaction between Gli1 and AQP1 in glioma metastasis. Our data suggested that both the mRNA and protein level of AQP1 and Gli1 expression are up-regulated in glioma tissue and in both two glioma cell lines, in comparison to normal control.

As mentioned above, Gli1 as a transcription factor can regulate the target gene expression via modulating its transcriptional state (21). In this content, it is worthy to note that our data provides directive evidence of Gli1 positively modulating the transcriptional expression of $A Q P 1$ gene via binding to $A Q P 1$ promotor. Therefore, we next evaluated whether the Gli1-AQP axis functions as internal molecular regulation mechanism underlying glioma cell survival, by assessing the cell viability and metastasis in presence of co-modulation of Gli1 and AQP1. We observed that knock down of Gli1 significantly reduced cell proliferation, migration and invasion of glioma cell. This anti-cell proliferation and metastatic effect of si-Gli1 was restored by AQP1 overexpression. This finding suggested a possible cellular interaction between Gli1-AQP signal transduction and the pathological process of gliomas, and also supported the view of potential therapeutic usefulness of combination of Gli1-targeted agents with standard treatments.
In summary, our study highlighted the evaluation of the Gli1-AQP1 axis in glioma cell survival for the first time, and we demonstrated that the internal molecular signal transduction of Gli1-AQP1 is involved in glioma survival. Our findings may provide new therapeutic targets for treatment of gliomas.

\section{MATERIALS AND METHODS}

\section{Clinical samples collection}

A total of 10 human glioma tissue were collected from surgical resection patients at The Nine People's Hospital of Chongqing, in the period between May 2013 to May 2014; the gliomas were confirmed by histopathologic examination. According to WHO pathological grades, two of tumor samples belonged to Grade III-IV. The rest were Grade IV. Three normal tissues, recognized as control, were obtained from intracranial decompression surgery.

This study obtained approval from the Ethical Committee of the Nine People's Hospital of Chongqing, and the patients gave written consent for tissue collection and analyses.

\section{Cell lines and culture}

Two human U251 and U87 glioma cell lines used in the in vitro experiments were obtained from the Shanghai Institute of Cell Biology, Chinese Academy of Sciences (CAS, Shanghai, China). Cells were incubated in high-glucose Dulbecco's Modified Eagle Medium (DMEM) (Life Technologies, Grand Island, NY, USA) supplemented with $10 \%$ fetal bovine serum, $100 \mu \mathrm{g} / \mathrm{mL}$ streptomycin (Invitrogen, Carlsbad, CA), and 100 $\mathrm{U} / \mathrm{mL}$ penicillin (HyClone, USA). Normal human astrocytes were obtained from Thermo Fisher Scientific (USA) and cultured in Gibco Astrocyte Medium (A1261301). All cells were cultured in a humidified incubator, at $37^{\circ} \mathrm{C}$ and $5 \% \mathrm{CO}_{2}$.

\section{mRNA Analysis in quantitative Real Time PCR (qRT-PCR)}

Total RNA was extracted from normal tissues and glioma cells using MagaZorb ${ }^{\circledR}$ Total RNA Mini-Prep Kit (Promega), according to the manufacturer's protocols. RNA was quantified using spectrophotometer. Equal amount of RNAs was reversed using RETROscript kit (Ambion, Life Technologies Invitrogen, Carlsbad, CA, USA), according to the manufacturer's protocols. To evaluate quantitative changes in mRNA levels of Gli1 and $A Q P 1$, each $R T$ reaction mixture was then subjected to real time PCR with Brilliant III Ultra-Fast SYBRH Green QPCR Master Mix (Stratagene, La Jolla, CA, USA). The PCR primers for detecting specific transcripts were as follows: Gli1, 5'-GCCAGCCAAGA GAGACCAACAG-3' and 5'-CCGACAGAGGTGAGATGGACA G-3'; AQP1, 5'-GAAGAAGCTCTTCTGGAGGGC-3' and 5'-AGC CAGGTCATTGCGGCCAAG-3'; $\beta$-actin, 5'-ACGTTGCTATCCAG GCTGTCCAT-3' and 5'-TTAATGTCACGCACGATTCCCGC-3'. Relative mRNA concentrations were calculated from the take-off point of reactions (threshold cycle, $\mathrm{Ct}$ ) using the comparative quantitation method based upon the $2^{\mathrm{DDCt}}$ method. Expression of $\beta$-actin was considered as internal reference. 


\section{Plasmid construction and transfection}

Gli1 was knocked down by cell transfected with pSuper-Gli1. In brief, 19bp reverse complement of middle of Gli1 cDNA (located at base site of 685-704) was inserted into the cohesive end of BgllI and HindIII. The constructor was collected by the boiling bath method, and then connected into pSuper vector, which we named as pSuper-Gli1. Positive clone was selected using the antibiotic kanamycin. The reverse sequences are as follows: Foward: 5'-GATCCCCAACTCCACAGGCATACAGGA TTTCAAGAGAATCCTGTATGCCTGTGGAGTTTTTTTA-3; reverse: 5'-AGCTTAAAAAAACTCCACAGGCATACAGGATTC TCTTGAAATCCTGTATGCCTGTGGAGTT-GGG-3'.

For Gli1 and AQP1 protein overexpression, plasmid containing Gli1 and AQP1 genes were constructed and transfected into glioma cells, respectively. In brief, Gli1 and AQP1 cDNA sequences were researched in $\mathrm{NCBI}$ and were amplified using PCR with glioma cell cDNA as template. The reaped cDNA was connected into pcDNA plasmid (Invitrogen, Carlsbad, CA, USA). The recombinant clones were screened using augmentin, and were named pcDNA-Gli1 and pcDNA-AQP1, respectively.

All recombinants were transfected into U251 or U87 cells using lipofectmine 2000 (Invitrogen, Shanghai, China), as per the manufacturer's protocols. Forty-eight hours post-transfection, the expression levels of Gli1 and AQP1, as well as cell viability and cell metastasis, were examined.

\section{Western blotting}

Western blot analysis was performed to determine protein levels of Gli1 and AQP1. Total proteins were extracted from tissue and cells using lysis buffer containing protease inhibitors from Calbiochem (California, USA). Soluble proteins were obtained by centrifugation, and concentration of the total protein was determined using BCA kit. For immunoblotting, equal amounts of protein were first separated by sodium dodecyl sulfate polyacrylamide gel electrophoresis (SDS-PAGE), and were then transferred to polyvinylidene fluoride (PVDF) membranes (Millipore, Billerica, MA, USA) using electrophoresis. The membrane was blocked by $5 \%$ skim milk powder (diluted in TBS) and was incubated with primary antibodies (Gli1; AQP; $\beta$-Actin). All antibodies were obtained from Abcam. The target proteins were then labeled by peroxidase-conjugated secondary antibodies (1:2000, Sigma Co., USA). The bands were visualized using ECL. Their intensity was analyzed using Scion image software (Frederick, MD).

\section{Dual-Luciferase reporter gene assay}

Glioma U251 and U87 cells were seeded at a density of $2 \times$ $10^{4}$ cells in 48-well plates. After $24 \mathrm{~h}$ incubation, the cells were grown to $60 \%-80 \%$ confluence, and were then co-transfected with pGL4-TK-Luc-AQP1, the control being pGL-SV40 and pcDNA-Gil1 or si-Gil1. After a $24 \mathrm{~h}$ transfection, cells were lysed and luminescence was analyzed using the dual-luciferase assay system (Promega). Relative Luciferase activity was normalized to pRL-SV40 activity.

\section{MTT assay}

Cell proliferation was evaluated by MTT assay. In brief, all glioma cell lines transfected with si-Gli1 or pcDNA-AQP1 were seeded in 48-well plates in complete culture medium, with the purpose of evaluation of cell proliferation by measurement of the dark blue formazan product of MTT (3-(4,5-Dimethylthiazol-2-yl)-2,5-diphenyltetrazolium bromide, a tetrazole). MTT was added to each well after $24 \mathrm{~h}$ of the beginning of the transfection, and incubated for $3 \mathrm{~h}$. The reaction was stopped by addition of the stop solution. Solubilization solution was then added to solubilize formazan product. Absorbance was analyzed in a 48-well plate reader, at $570 \mathrm{~nm}$.

\section{Cell migration assays}

Glioma cell migration ability was assessed using 24-well transwell plates $(8.0-\mu \mathrm{m}$ pore membranes; BD Biosciences, CA, USA). In brief, about $1 \times 10^{4}$ cells were loaded into the upper chambers of the transwell, and the lower chambers were filled with medium (plus $1 \% \mathrm{FBS}$ ). After $48 \mathrm{~h}$ incubation at $37^{\circ} \mathrm{C}$ and $5 \% \mathrm{CO}_{2}$, the cells were harvested in the upper side of polycarbonate membrane of transwell plates, and washed by PBS buffer. The washed cells in polycarbonate membrane were then stained by hematoxylin-eosin, and microscopically evaluated. The total migrated cells were photographed, their percentage calculated.

\section{Matrigel invasion assay}

Matrigel glioma cell invasion was assessed using the BD Biocoat Matrigel Invasion Chamber (pore size: $8 \mathrm{~mm}$, 24-well; BD Biosciences, CA, USA). In brief, about $5 \times 10^{4}$ cells were plated in the upper chamber of transwell in serum-free medium containing $1 \mathrm{mg} / \mathrm{ml}$ matrigel; the bottom chamber contained medium with $10 \%$ FBS. After $48 \mathrm{~h}$ incubation at $37^{\circ} \mathrm{C}$ and $5 \% \mathrm{CO}_{2}$, the cells in the bottom of the chamber insert were stained with Calcein AM (Invitrogen, Carlsbad, CA, USA). The cells that had invaded through the membrane to the lower surface were evaluated in a fluorescence plate reader at excitation/emission of $492 \mathrm{~nm} / 515 \mathrm{~nm}$.

\section{ChIP assay}

The possible target AQP1 gene by Gli1 was determined using the ChIP assay. In brief, cells were cross-linked with $1 \%$ formaldehyde, following which they were broken using ultrasonication. Soluble chromatin from the broken cells was collected by centrifugation, and incubated overnight at $4^{\circ} \mathrm{C}$ with antibodies against Gli1 (Santa Cruz Biotechnology, Inc.) for immunoprecipitation; immunoprecipitation with equal rabbit IgG was used as the negative control. After washing, and DNA sample was obtained from the immune complex by Gel Extraction Kit (Omega Bio-tek, GA, USA). The recovered DNA was then analyzed by qRT-PCR using primers flanking the ARE of the AQP1 promoter. Results were normalized to input controls. 


\section{Statistical analysis}

All data are expressed as mean \pm standard deviation (SD). The Student's t-test or one-way analysis of variance was used to compare differential significance with control value using SPSS 13.0 software (SPSS Inc., Chicago, IL, USA). A P value less than $<0.05$ was considered to be statistically significant.

\section{REFERENCES}

1. Cordier N, Delingette $\mathrm{H}$ and Ayache N (2016) A patch-based approach for the segmentation of pathologies: Application to glioma labelling. IEEE Trans Med Imaging 35, 1066-1076

2. Molinaro AM, Wrensch MR, Jenkins RB and Eckel-Passow JE (2016) Statistical considerations on prognostic models for glioma. Neuro Oncol 18, 609-623

3. Roder C, Bisdas S, Ebner FH et al (2014) Maximizing the extent of resection and survival benefit of patients in glioblastoma surgery: high-field iMRI versus conventional and 5-ALA-assisted surgery. Eur J Surg Oncol 40, 297-304

4. Quail DF and Joyce JA (2013) Microenvironmental regulation of tumor progression and metastasis. Nat Med $19,1423-1437$

5. Hambardzumyan D, Gutmann DH and Kettenmann $\mathrm{H}$ (2015) The role of microglia and macrophages in glioma maintenance and progression. Nat Neurosci 19, 20-27

6. Liu X, Wang X, Du W et al (2014) Suppressor of fused (Sufu) represses Gli1 transcription and nuclear accumulation, inhibits glioma cell proliferation, invasion and vasculogenic mimicry, improving glioma chemo-sensitivity and prognosis. Oncotarget 5, 11681-11694

7. Du WZ, Feng Y, Wang XF et al (2013) Curcumin suppresses malignant glioma cells growth and induces apoptosis by inhibition of SHH/GLI1 signaling pathway in vitro and vivo. CNS Neurosci Ther 19, 926-936

8. Ji H, Miao J, Zhang X et al (2012) Inhibition of sonic hedgehog signaling aggravates brain damage associated with the down-regulation of Gli1, Ptch1 and SOD1 expression in acute ischemic stroke. Neurosci Lett 506, $1-6$

9. Wang LW, Lin H, Lu Y, Xia W, Gao J and Li ZS (2014) Sonic hedgehog expression in a rat model of chronic pancreatitis. World J Gastroenterol 20, 4712-4717

10. Wick W and Kuker W (2004) Brain edema in neurooncology: radiological assessment and management.
Onkologie 27, 261-266

11. El Hindy N, Rump K, Lambertz N et al (2013) The functional Aquaporin $1-783 \mathrm{G} / \mathrm{C}-$ polymorphism is associated with survival in patients with glioblastoma multiforme. J Surg Oncol 108, 492-498

12. Deb $\mathrm{P}$, Pal $\mathrm{S}$, Dutta $\mathrm{V}$, Boruah $\mathrm{D}$, Chandran VM and Bhatoe HS (2012) Correlation of expression pattern of aquaporin-1 in primary central nervous system tumors with tumor type, grade, proliferation, microvessel density, contrast-enhancement and perilesional edema. J Cancer Res Ther 8, 571-577

13. Qiu B, Li X, Sun X et al (2014) Overexpression of aquaporin1 aggravates hippocampal damage in mouse traumatic brain injury models. Mol Med Rep 9, 916-922

14. Park M, Song C, Yoon H and Choi KH (2016) Double Blockade of Glioma Cell Proliferation and Migration by Temozolomide Conjugated with NPPB, a Chloride Channel Blocker. ACS Chem Neurosci 7, 275-285

15. Carpenter RL, Paw I, Zhu H et al (2015) The gain-offunction GLI1 transcription factor TGLI1 enhances expression of VEGF-C and TEM7 to promote glioblastoma angiogenesis. Oncotarget 6, 22653-22665

16. Lampichler K, Ferrer P, Vila G et al (2015) The role of proto-oncogene GLI1 in pituitary adenoma formation and cell survival regulation. Endocr Relat Cancer 22, 793-803

17. Singh RR, Kunkalla K, Qu C et al (2011) ABCG2 is a direct transcriptional target of hedgehog signaling and involved in stroma-induced drug tolerance in diffuse large B-cell lymphoma. Oncogene 30, 4874-4886

18. Liu S, Duan X, Xu L et al (2016) Nuclear Gli1 expression is associated with pathological complete response and event-free survival in HER2-positive breast cancer treated with trastuzumab-based neoadjuvant therapy. Tumour Biol 37, 4873-4881

19. Morrissey JJ, Mellnick VM, Luo J et al (2015) Evaluation of Urine Aquaporin-1 and Perilipin-2 Concentrations as Biomarkers to Screen for Renal Cell Carcinoma: A Prospective Cohort Study. JAMA Oncol 1, 204-212

20. Wei X and Dong J (2015) Aquaporin 1 promotes the proliferation and migration of lung cancer cell in vitro. Oncol Rep 34, 1440-1448

21. Bora-Singhal N, Perumal D, Nguyen J and Chellappan $S$ (2015) Gli1-Mediated Regulation of Sox2 Facilitates Self-Renewal of Stem-Like Cells and Confers Resistance to EGFR Inhibitors in Non-Small Cell Lung Cancer. Neoplasia 17, 538-551 